\title{
Spatial Variation of Unit Hydrograph Parameters for Rainfall Derived Infiltration/Inflow and the Relationship with Physical Factors
}

\section{Li Zhang, Fang Cheng, Gregory Barden, Hunter Kelly, Timothy Fallara and Edward Burgess}

This chapter presents a statistical analysis of the spatial variation of total runoff and initial abstraction (IA), and their relationship with physical system factors. The work was performed for the City of Columbus Sewer System Capacity Model Update 2006 (SSCM 2006) project in Columbus, Ohio. The USEPA's SWMM5 program provides a synthetic unit hydrograph (SUH) approach to characterize the rainfall derived infiltration and inflow (RDII) response to a rainfall event using unit hydrograph parameters (RTK) and the IA parameters.

Total runoff and IA were obtained from a continuous model calibrated with seasonal (dormant and growth seasons) RTK and seasonal IA parameters to simulate RDII using SWMM5. Global Moran's I and Anselin local Moran's I tests were performed on seasonal total runoff and IA to detect spatial autocorrelation and clusters and outliers. Multivariate regression analysis was performed to evaluate the relationship of total runoff and IA with various physical system factors.

The results showed significant spatial autocorrelation of both total runoff and IA for both seasons. $R$ for the unit hydrograph and $D_{\max }$ for the IA parameters were used in the spatial variation and regression analysis as response

Zhang, L., F. Cheng, G. Barden, H. Kelly, T. Fallara and E. Burgess. 2013. "Spatial Variation of Unit Hydrograph Parameters for Rainfall Derived Infiltration/Inflow and the Relationship with Physical Factors." Journal of Water Management Modeling R246-04. doi: 10.14796/JWMM.R246-04.

(C) CHI 2013 www.chijournal.org ISSN: 2292-6062 (Formerly in Pragmatic Modeling of Urban Water Systems. ISBN: 978-0-9808853-8-5) 
variables. $R$ represents the fraction of rainfall volume that becomes inflow or infiltration in the collection system. $D_{\max }$ is the maximum possible depth in soil ground available for IA. Regression analysis of $R$ and $D_{\max }$ with the physical factors revealed a strong relationship of total runoff with pipe density and pipe age. The significant relationship found in this study has benefitted the project as a means of estimating total runoff for basins which cannot be calibrated directly.

\subsection{Introduction}

Rainfall derived infiltration and inflow into sanitary sewers is known to be a major contributor to sanitary sewer overflow occurrences and water-inbasement (WIB) complaints. The modeling of sanitary sewer systems is commonly employed to investigate these problems, using continuous or single event simulations. Continuous simulation can simulate RDII more effectively than single event simulations by incorporating antecedent moisture conditions (AMC) directly, rather than using assumed AMC as necessary for single event simulations.

AMC are represented by monthly IA parameters in SWMM5, which is used with the unit hydrograph parameters (RTK) to continuously simulate RDII. Monitoring limitations often hinder the accurate calibration of these parameters, and assumed values need to be used. Understanding both the spatial and temporal variations of the empirically derived unit hydrograph parameters, including both total RDII capture fractions (total runoff and IA), is important to accurately establish values for these model parameters to obtain robust simulation results.

This chapter presents a statistical analysis of spatial variation of total runoff and IA and their relationship with physical system factors, as part of the Sewer System Capacity Model Update 2006 project for the City of Columbus, Ohio. Total runoff and IA were obtained from continuous calibration of the system wide model. Global Moran's I and Anselin local Moran's I tests were performed on seasonal $R$ and IA to detect spatial autocorrelation and clusters and outliers. Multivariate regression analysis was performed to evaluate the relationship of $R$ and IA with various physical system factors.

\subsection{Background}

\subsubsection{Study Area}

To understand the flow characteristics in the system, more than 100 flow monitors were installed and continuously monitored for sixteen months from 
January 2008 to May 2009. Radar rainfall data were available for eighteen relatively large events during the monitoring period. The data of eighteen radar rainfall storms were amalgamated with data from 30 rain gauges to provide continuous rainfall data for the same period. The flow meter data and rainfall data were used for the continuous simulation of wet weather flow, including RDII quantification.

\subsubsection{RDII Analysis in SWMM5}

SWMM5 provides a synthetic unit hydrograph (SUH) approach to characterize the RDII response to a rainfall event. The RDII response is simulated using eighteen parameters on a monthly basis. Figure 4.1 and Figure 4.2 show the SUH settings in SWMM5. The approach uses three sets of parameters to define the short term, intermediate term and long term response of inflow and infiltration. Each set includes 6 parameters which are $R, T$ and $K$ for the unit hydrograph and $D_{\max }, D_{\text {rec }}$ and $D_{0}$ for the initial abstraction (IA). $R$ is the fraction of rainfall volume that enters the sewer system, $T$ is the time from the onset of rainfall to the peak of the $\mathrm{SUH}$, and $K$ is the ratio of time-to-recession to the time-to-peak of the unit hydrograph. $D_{\max }$ is a maximum possible depth of IA, $D_{r e c}$ is a recovery rate at which stored IA is depleted during dry periods, and $D_{0}$ is an initial depth of stored IA at the start of the simulation (USEPA, 2009).

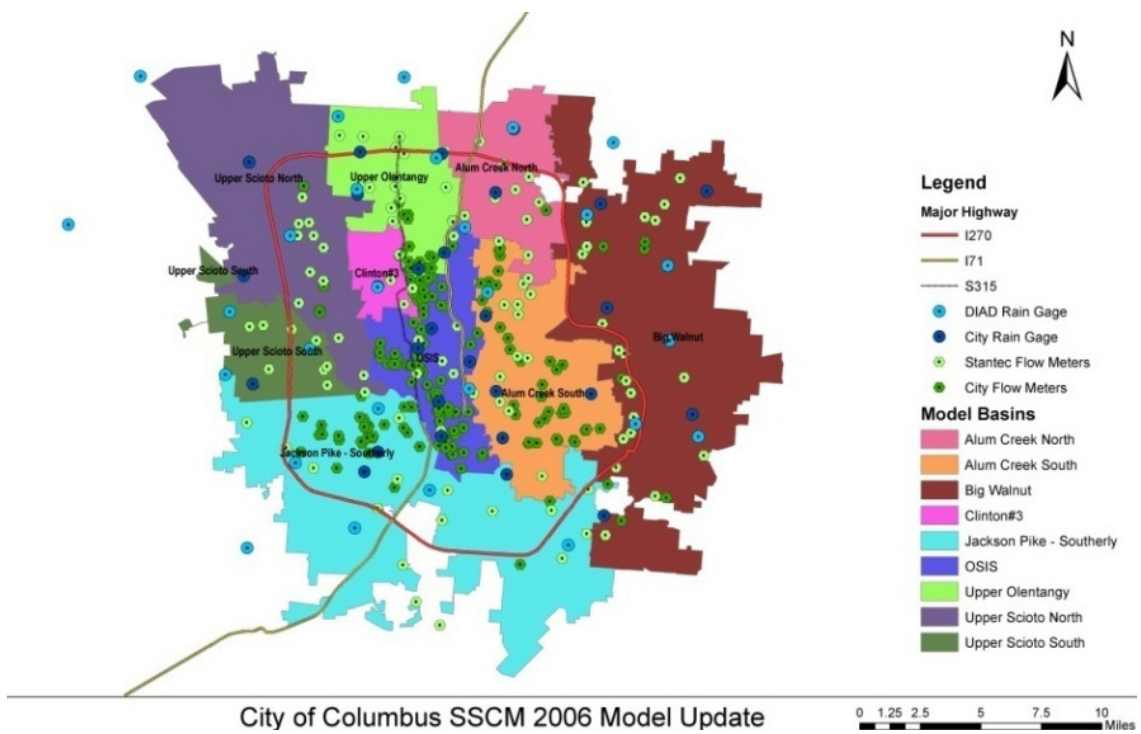

Figure 4.1 Flow meters and rain gauges in Columbus. 


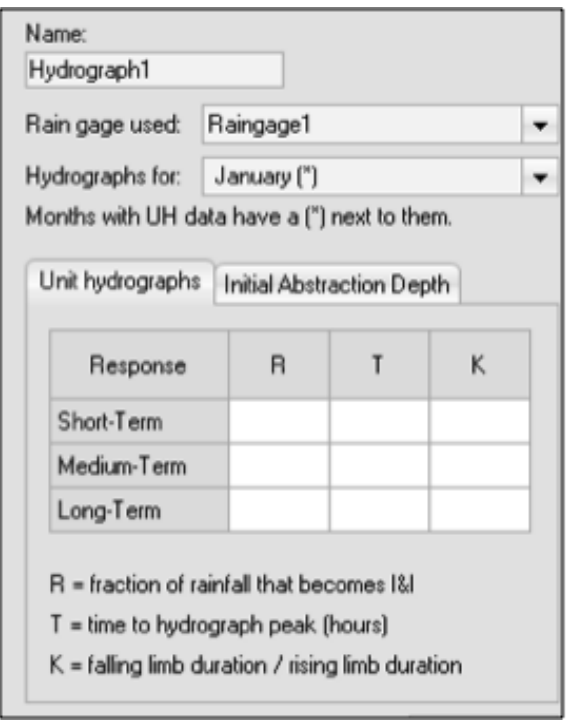

\begin{tabular}{|c|c|c|c|c|}
\hline \multicolumn{5}{|l|}{ Name: } \\
\hline \multicolumn{5}{|l|}{ Hydrograph1 } \\
\hline \multirow{2}{*}{$\begin{array}{l}\text { Rain gage used: } \\
\text { Hydrographs for: }\end{array}$} & \multicolumn{3}{|l|}{ Raingage1 } & $\checkmark$ \\
\hline & \multicolumn{3}{|l|}{ January (") } & $\boldsymbol{\nabla}$ \\
\hline \multicolumn{5}{|c|}{ Months with UH data have a (") next to them. } \\
\hline Unit hydrographs & \multicolumn{3}{|c|}{ Initial Abstraction Depth } & \\
\hline Response & $D \max$ & Drec & Do & \\
\hline \multicolumn{5}{|l|}{ Short-Term } \\
\hline \multicolumn{5}{|l|}{ Medium-Term } \\
\hline \multicolumn{5}{|l|}{ Long-Term } \\
\hline \multicolumn{5}{|c|}{ Dmax $=$ maximum depth [inches] } \\
\hline \multicolumn{5}{|c|}{ Drec $=$ recovery rate $[\mathrm{n} /$ day $]$} \\
\hline \multicolumn{5}{|c|}{$D_{0}=$ starting depth [inches] } \\
\hline
\end{tabular}

Figure 4.2 RTK and IA for synthetic unit hydrograph in SWMM5.

The RTK parameters define the shape of the unit hydrograph, and the IA parameters represent the antecedent moisture conditions. $R$ represents the fraction of rainfall volume that becomes inflow or infiltration in the collection system. $T$ is the time from the onset of rainfall to the peak of the unit hydrograph. $K$ is the ratio of time-to-recession of the unit hydrograph to the parameter $T . D_{\max }$ is the maximum possible depth of initial abstraction (i.e. the maximum storage in soil available for initial abstraction). $D_{\text {rec }}$ is the recovery rate at which stored initial abstraction decayed during dry periods. $D_{0}$ is the initial depth of stored initial abstraction which is only utilized at the beginning of the simulation.

Initial abstraction represents the amount of rainfall absorbed by soil ground which does not produce any I/I response. When initial abstraction parameters were used with the unit hydrograph parameters (RTK) to continuously simulate RDII, the rainfall volume to produce RDII would be the total rainfall volume with the initial abstraction subtracted from it.

\subsubsection{Continuous Calibration}

Continuous calibration was performed for the SSCM Model Update project for the period of January 2008 to May 2009 using PCSWMM (CHI, 2010) with the USEPA's SWMM5 as the computational engine. RDII response at a 
given area may be affected by seasonal factors such as vegetation coverage, groundwater table, temperature, and evaporation rate (Zhang et al., 2010). Antecedent moisture conditions may also vary with those seasonal factors. Because of the lack of long term (i.e. multiple years) monitoring on finer spatial scale, seasonal parameters were considered instead of using monthly SUH parameters. Study in the Columbus area showed a significant seasonal variation of RDII response (Cheng et al., 2010). The same amount of rainfall produces much higher RDII in the dormant season than it does in the growth season over the same area. Models with seasonal RTK and seasonal IA performed relatively well for moderate to large RDII responses (Cheng et al., 2010). Accordingly, seasonal parameters were used for the RDII hydrograph for the calibration of the continuous simulation model for the SSCM MU 2006 project. The months from January to April were defined as the dormant season, and the months from May to December were defined as the growth season. More than 100 flow meter basins were calibrated. The calibrated seasonal RTK and IA parameters were used for the spatial variation analysis in this study.

\subsection{Approach and Results}

\subsubsection{Spatial Variation Analysis}

To understand the spatial variation of RDII, Moran's I tests were performed on the total runoff and IA of the calibrated SUH parameters from the continuous simulation model. The SUH hydrograph contains three sets of RTK and IA for the short term, medium term and long term RDII responses to rainfall. In this study, the sum of the three $R$ values and the average of the three $D_{\max }$ values of IA were used for analyzing the spatial variation in both the dormant and growth seasons.

Spatial autocorrelation is characterized by a correlation of an attribute of a feature with the attributes of features at nearby locations in space. In this study, the features in space are the flow meter basins calibrated in the continuous model. The attributes are the total runoff and the average IA for the spatial variation analysis.

Moran's I test was developed by Patrick A. P. Moran to measure spatial autocorrelation (Moran, 1950). The test evaluates whether the spatial pattern for that attribute is clustered, dispersed or random, given a set of features (in this study, flow meter basins), and an associated attribute (total runoff or average IA). Moran's I is an index that is a measure of feature similarity based on both feature locations and feature values simultaneously. The calculation of 
Moran's I index is:

$$
I=\frac{n \sum_{i=1}^{n} \sum_{j=1}^{n} w_{i, j}\left(x_{i}-\bar{x}\right)\left(x_{j}-\bar{x}\right)}{S_{0} \sum_{i=1}^{n}\left(x_{i}-\bar{x}\right)^{2}}
$$

where:

$x_{i}=$ attribute of feature $i$,

$n=$ the total number of features,

$\bar{x}=$ the mean of the corresponding attribute of all the $n$ features,

$w_{i, j}=$ the weight between observation $i$ and $j$, and where:

$S_{0}=$ the sum of all $w_{i, j}$

$$
S_{0}=\sum_{i=1}^{n} \sum_{j=1}^{n} w_{i, j}
$$

Moran's I provides global spatial autocorrelation analysis with only one statistic to summarize the whole study area. Having only one statistic does not provide enough information when that statistic may differ over space. Local spatial autocorrelation statistics can provide a measure, for each feature in the space, of the tendency to have an attribute value that is correlated with the attribute values of the features nearby. Anselin local Moran's I (Anselin, 1995) identifies clusters of features with values similar in magnitude, and also features with values distinctive from surrounding features, as spatial outliers. The local Moran's I value is calculated as:

$$
I_{i}=\frac{\left(x_{i}-\bar{x}\right) \sum_{j=1, j \neq i}^{n} w_{i, j}\left(x_{j}-\bar{x}\right)}{S_{i}^{2}}
$$

where:

$x_{i}=$ attribute of feature $i$,

$n=$ total number of features,

$\bar{x}=$ mean of the corresponding attribute of all the $n$ features,

$w_{i, j}=$ weight between observation $i$ and $j$, and

$$
S_{i}^{2}=\frac{\sum_{j=1, j \neq i}^{n} w_{i, j}}{n-1}
$$

A high positive value for Moran's I indicates a clustered pattern of the 
attribute in space, while a high negative value for Moran's I indicates a dispersed pattern. The significance of Moran's I statistics can be measured by $p$-values or $z$-scores; $p$-value is the probability to falsely reject the null hypothesis of random distribution in space and $z$-score is a measure of standard deviation. Very high or very low (negative) $z$-scores, associated with very small $p$-values, are an indication of a low possibility that the distribution of the attribute are random in space. Both statistics are associated with the standard normal distribution (ArcGIS, 2009).

Moran's I test results on both the runoff and IA on dormant and growth seasons were summarized in Table 4.1. The $p$-values from the four tests on $R$ and IA in both seasons were less than 0.01 , indicating that there is $<1 \%$ possibility that the pattern of IA and runoff were from random spatial data.

Table 4.1 Results of Moran's I tests on dormant and growth R and IA.

\begin{tabular}{lcccc}
\hline & Dormant $R$ & Growth $R$ & Dormant IA & Growth IA \\
\hline Moran's I & 0.51 & 0.63 & 0.29 & 0.26 \\
$z$-score & 19.07 & 23.29 & 10.87 & 9.89 \\
$p$-value & $<0.01$ & $<0.01$ & $<0.01$ & $<0.01$ \\
\hline
\end{tabular}

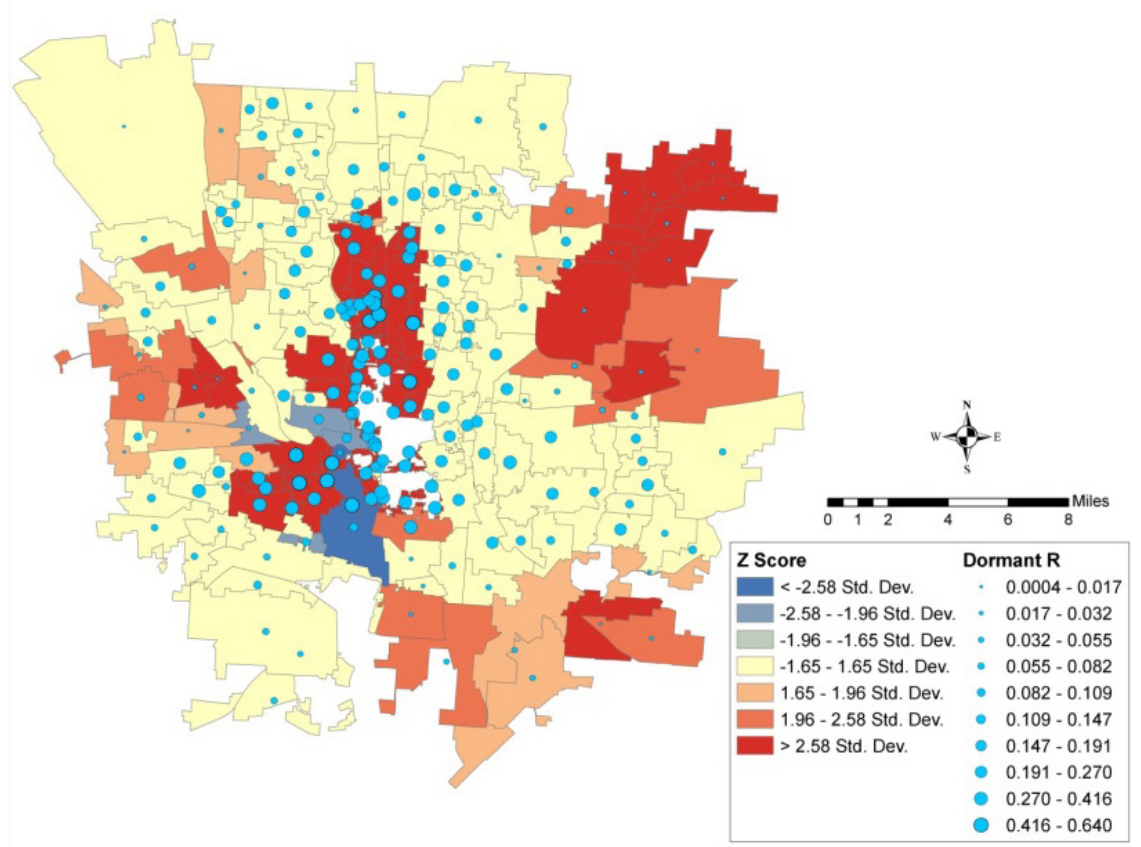

Figure 4.3 Result of local Moran's I test on dormant $R$. 


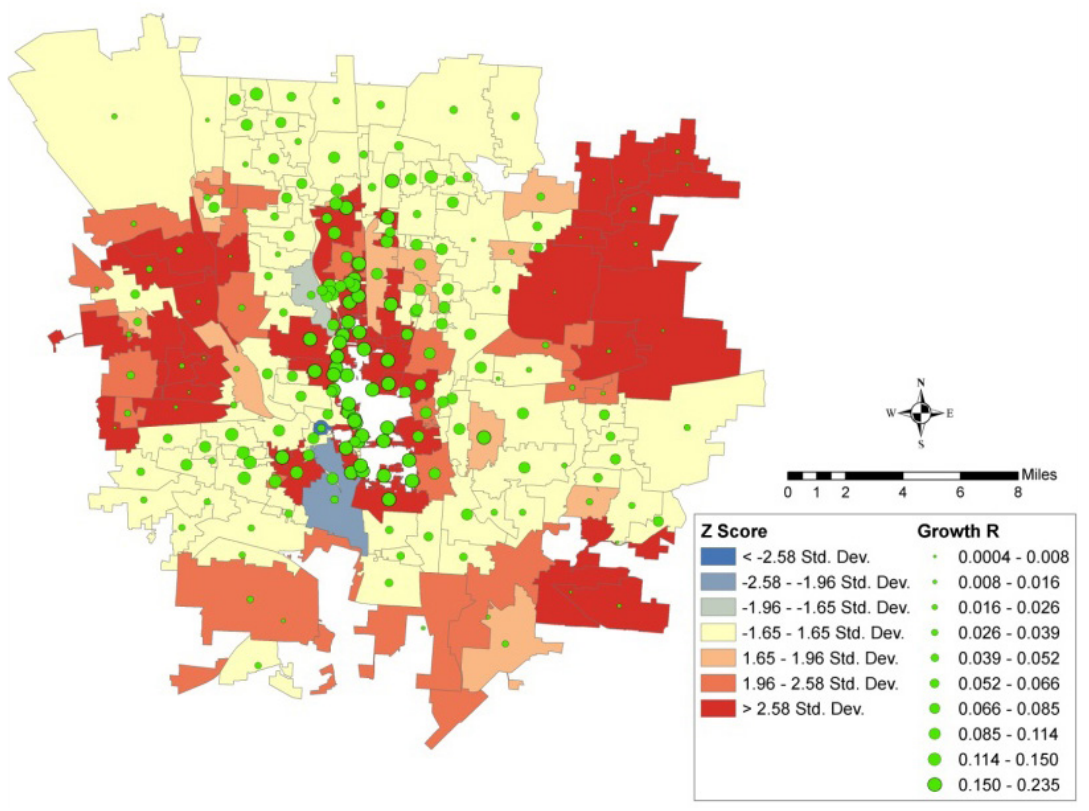

Figure 4.4 Result of local Moran's I test on growth $R$.

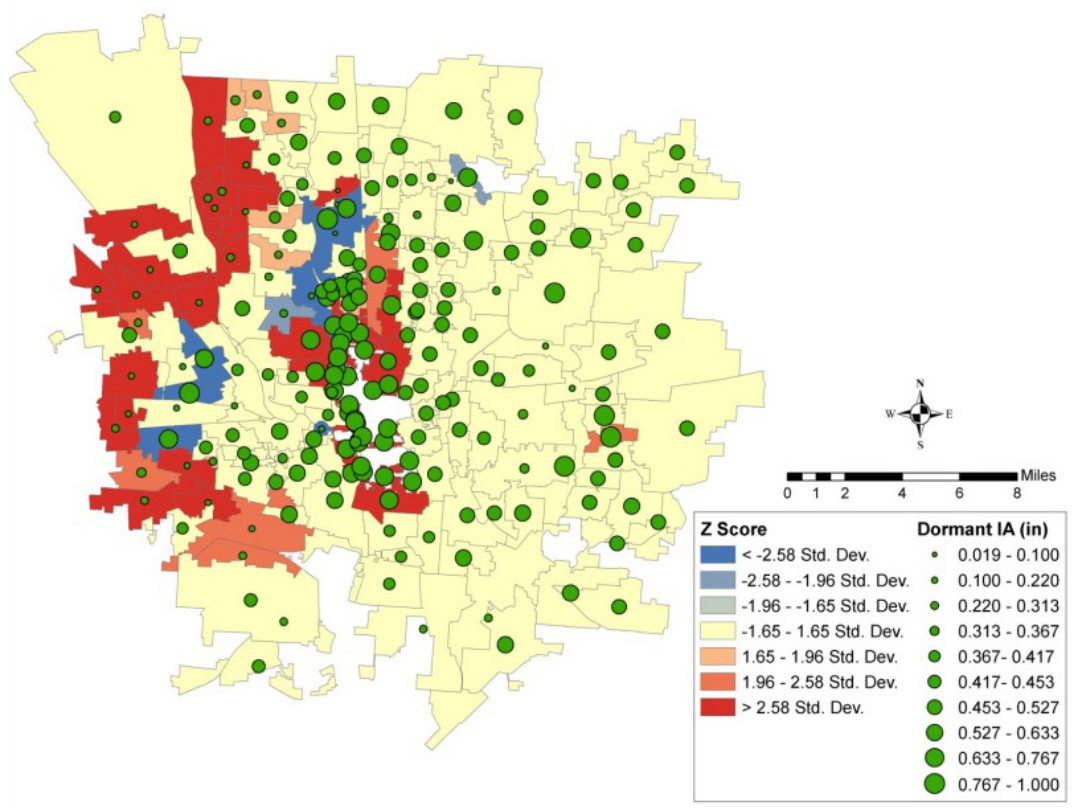

Figure 4.5 Result of local Moran's I test on dormant IA. 


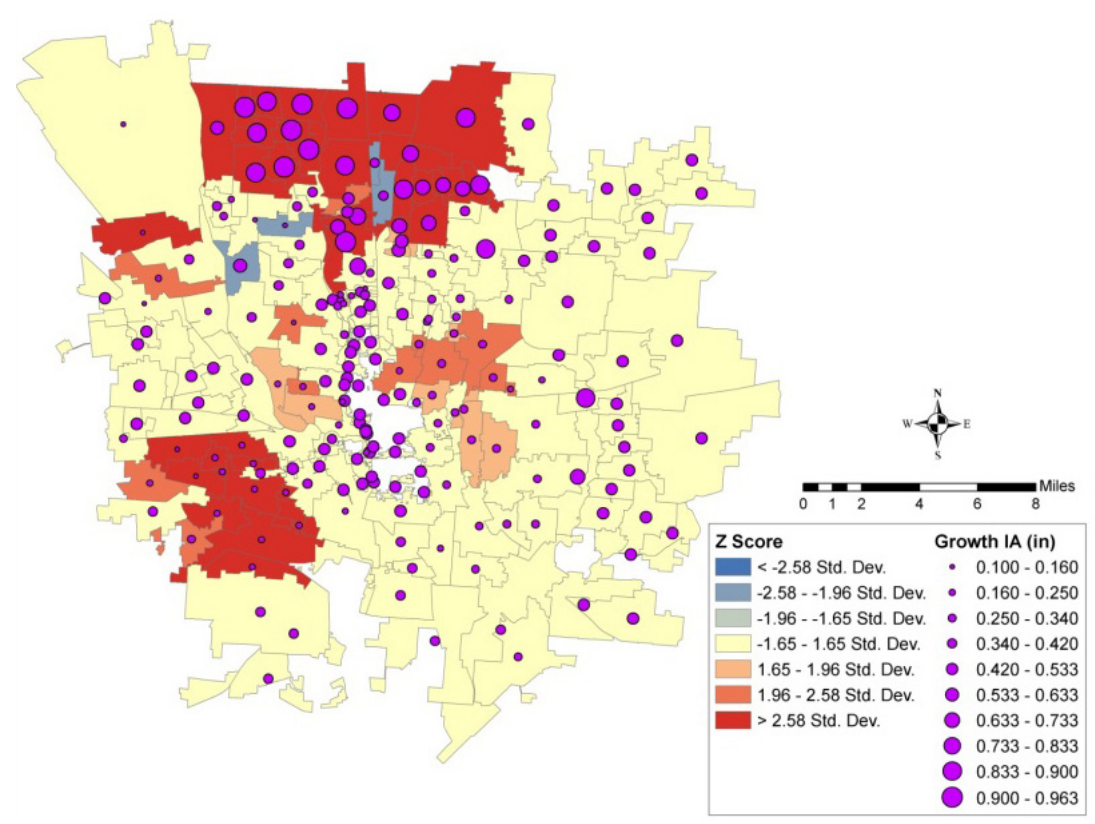

Figure 4.6 Result of local Moran's I test on growth IA.

The results of the $z$-scores of local Moran's I tests were demonstrated in Figure 4.3 through Figure 4.6 above. A high positive value for the $z$-score indicates that the feature is surrounded by features with similar values as part of a cluster. A high negative $z$-score value indicates that the feature is surrounded by features with dissimilar values as an outlier. Figure 4.3 and Figure 4.4 show similar patterns of clusters and outliers of total runoff in dormant and growth seasons. Figure 4.5 and Figure 4.6 show similar patterns of clusters and outliers of average IA in dormant and growth seasons.

\subsubsection{Regression Analysis}

For a given flow meter basin area, the RDII response may be affected by physical system factors such as pipe density (length of pipes/area), pipe age, land use, vegetation coverage or soil type. To understand the relationship, regression analyses were performed on the total runoff and the average IA using the physical factors as candidate predictors. In this study, two physical factors including pipe density and pipe age were used in the regression analysis while other factors were not analyzed due to the limitation of time and the availability of data. 
The average pipe age was defined as the average of the age of all the pipes in the flow meter basins (the difference between 2010 from the year the pipe was built). The pipe density was defined as the total length of pipes in the flow meter basins divided by the total basin area.

Scatter plots in Figure 4. 7 and Figure 4.8 opposite show the relationships of the pipe age and the pipe density with the average IA or the total runoff in the dormant and growth seasons. The total runoff showed an obvious positive relationship with both pipe age and pipe density, while the average IA showed no obvious relationship with pipe age or pipe density

The result of the regression equation of the total runoff in dormant season on the pipe age and pipe density is:

$$
\sqrt{R_{D r}}=-0.439+0.179 * \operatorname{Ln}(\text { Age })+0.00166 * \text { Density }
$$

The result of the regression equation of the total $\mathrm{R}$ in growth season on the pipe age and pipe density is:

$$
\sqrt{R_{G r}}=-0.247+0.108 * \operatorname{Ln}(\text { Age })+0.00110 * \text { Density }
$$

where:

$R_{D r}=$ total runoff for the dormant season,

$R_{G r}=$ total runoff for the growth season,

Age $=$ average age for all pipes in each flow meter basin, and

Density $=$ ratio of total pipe length to the area of each flow meter basin.

The coefficient of multiple determination $R^{2}$ is a statistical parameter to measure the accuracy of a given regression equation. The adjusted $R^{2}$ is a modified measure given by adjusting for the number of predictors in the model, as $R^{2}$ is increased and never decreased by adding more predictors (Kutner et al., 2004).

The adjusted $R^{2}$ of the regression of the total runoff in the dormant season is $74.8 \%$. The adjusted $R^{2}$ of the regression of the total runoff in the growth season is $73.0 \%$. These indicate that more than $70 \%$ of the variation of the total runoff can be explained by the pipe age and pipe density. Regression of both seasons showed a significant relationship between the total runoff and the pipe age and pipe density. The regression was also performed on the average IA using the pipe age and pipe density; in this case however, no significant relationship were found. 


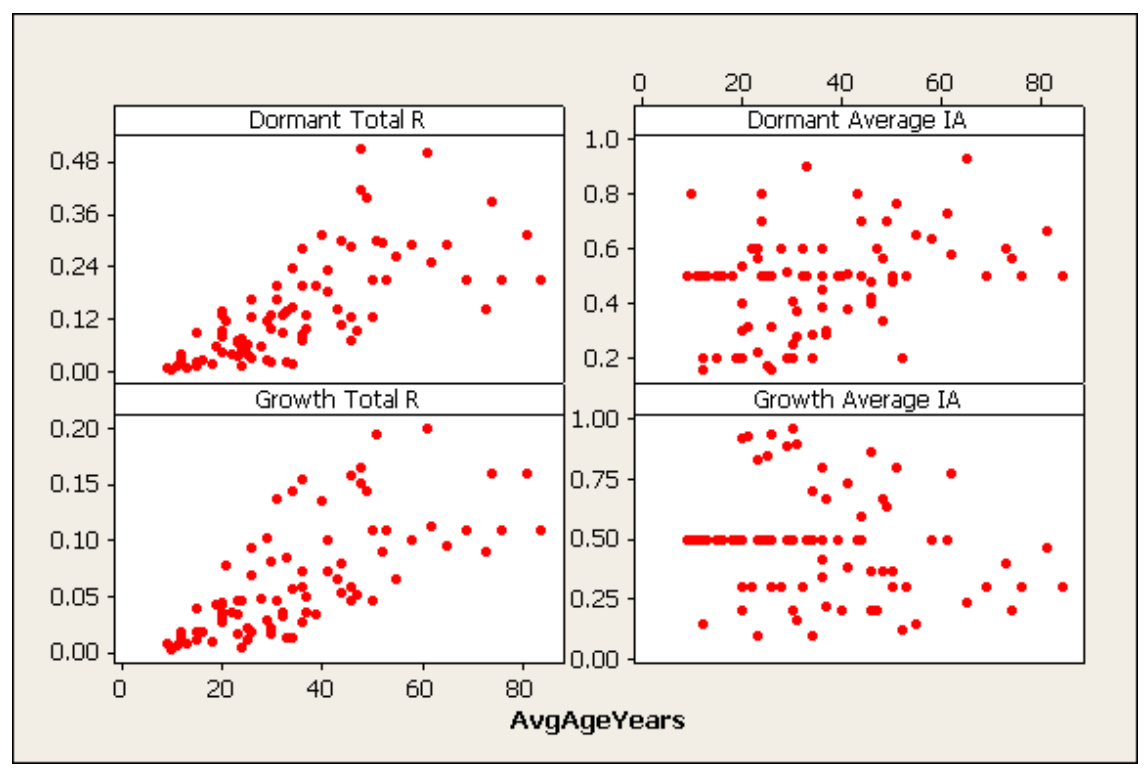

Figure 4.7 Pipe age vs runoff and IA in dormant and growth seasons.

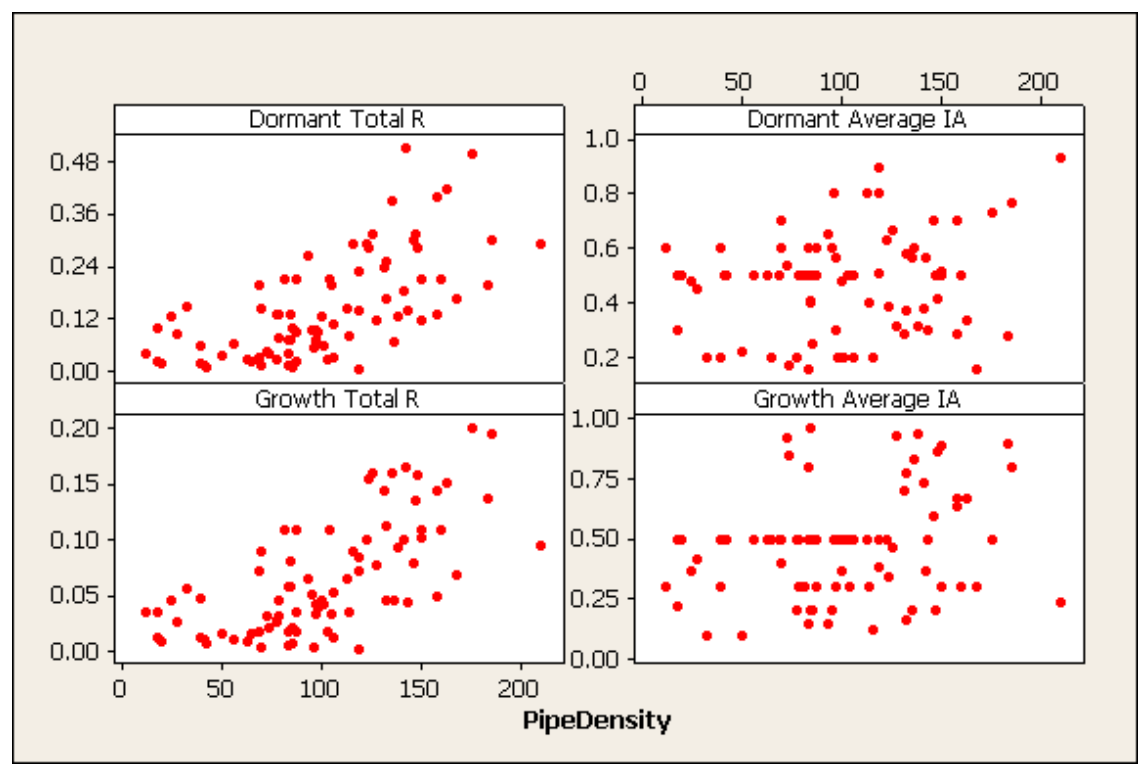

Figure 4.8 Pipe density vs runoff and IA in dormant and growth seasons. 


\subsection{Discussion}

The significant relationship of autocorrelation of $R$ and IA in both dormant and growth seasons supports the estimation of the SUH parameters for the RDII response for a given basin using the surrounding basins. The regression results of total runoff with the pipe age and pipe density indicate a basin with similar pipe age and pipe density in the Columbus area may have a similar total runoff value for RDII responses. The significant relationship found in this study has benefited Columbus's SSCM 2006 model, as a means of estimating total runoff for basins which were not monitored or cannot be calibrated directly. The study has shown that the calibrated basin nearby or downstream basins with similar pipe density and pipe age may provide similar RDII responses. Therefore, the RTK and IA of the non-monitored basins can be estimated based on the nearby basins. Further testing on other systems may reveal whether this finding is broadly applicable or only applies to Columbus, Ohio. More analysis is needed for the relationship of RDII response with the other physical factors such as land use, vegetation coverage or soil type.

\subsection{Conclusions}

Significant spatial autocorrelation of the total runoff and the average IA (the SWMM5 $D_{\max }$ parameter) were found for both dormant and growth seasons. The $p$-values from the spatial autocorrelation tests on both total runoff and IA were $<0.01$ for both the seasons, indicating that there is $<1 \%$ possibility that the spatial patterns of IA and total runoff were from random spatial data. The total runoff showed more significant spatial autocorrelation than the average $D_{\max }$. Regression analysis was performed to analyze the relationship of the total runoff and the average $D_{\max }$ with the physical factors including both pipe density and pipe age. Results revealed a strong positive relationship of the total runoff with the pipe age and the pipe density in both the dormant and growth seasons. The values of the adjusted $R^{2}$ for both seasons are over $70 \%$, which indicates that more than $70 \%$ of the varation of the total runoff can be explained by the pipe age and the pipe density. However, no significant relationship was detected between the average $D_{\max }$ and the pipe age and the pipe density. The significant relationship identified in this paper is useful as a means of estimating total runoff for basins that cannot be calibrated directly.

\section{Acknowledgment}

This study was supported by the City of Columbus, Ohio Sewer System Capacity Model Update 2006 project (SSCM, 2006). 


\section{References}

Anselin, L. (1995). Local Indicators of Spatial Association -LISA, Geographical Analysis, 27(2), p. 93-115.

ArcGIS (2009). Spatial Statistics toolbox, Geoprocessing tool reference.

Cheng, F., B.J. Sherman, G. Barden, H. Kelly, T. Fallara and E. Burgess. 2010.

"Comparison of RDII Unit Hydrograph Approaches for Continuous Simulation using SWMM 5." Journal of Water Management Modeling R241-12. doi: 10.14796/JWMM.R241-12.

CHI (2010). PCSWMM 2010 user documentation. Computational Hydraulics Int., Guelph Ontario, Canada

USEPA (2009). Storm Water Management Model User's Manual Version 5.0., U.S. EPA/600/R-05/040.

Moran, P.A.P. (1950). Notes on Continuous Stochastic Phenomena, Biometrika, Jun., 1950, vol. 37, no. 1/2, p. 17-23.

Kutner, M. H., Nachtsheim, C. J. and Neter, J. (2004). Applied Linear Regression Models, Fourth Edition, McGraw-Hill/Irwin, New York.

Zhang, L., F. Cheng, G. Barden, H. Kelly, T. Fallara and E. Burgess. 2010. "Regression Analysis of the Variation in Rainfall Derived Inflow and Infiltration." Journal of Water Management Modeling R241-13. doi: 10.14796/JWMM.R241-13. 
